\title{
Research Paper \\ The Impact of Rehabilitation Module of Iran Welfare Organization among Older Adults
}

\author{
Mohammad Nasiri ${ }^{1}$, Mahshid Foroughan², Vahid Rashedi², Mohammad Reza Shahbazi²
}

1. Deparment of Rehabilitation Administration, University of Social Welfare and Rehabilitation Sciences, Tehran, Iran.

2. Iranian Research Center on Ageing, University of Social Welfare and Rehabilitation Sciences, Tehran, Iran.

Cltation: Nasiri M, Foroughan M, Rashedi V, Shahbazi MR. [The Impact of Rehabilitation Module of Iran Welfare Organization among Older Adults (Persian)]. Iranian Journal of Ageing. 2016; 11(4):110-117. http://dx.doi.org/10.21859/sija-1101110

: http://dx.doi.org/10.21859/sija-1101110

Received: 04 Oct. 2015 Accepted: 08 Feb. 2016

\begin{abstract}
A B STRACT
Objectives With the increasing aging population, attention to their problems in society has found vast and new dimensions. The professional rehabilitation teams in day-care centers have aimed at providing services to the elderly to prevent their admission into residential boarding centers. To this end, the Welfare Organization has designed a special service package for the day-care centers. The main objective of this study was to compare the quality of life of older adults in a day center before and after receiving the rehabilitation module of the Welfare Organization.

Methods \& Materials This is a descriptive-analytic study with 2 groups and pretest/posttest design. The study population consisted of all older adults referred to day-care centers for older adults. The study sample comprised 50 older adults in the Kahrizak Rehabilitation Center No.2, located in Mohammad Shahr Karaj, Iran in 2014-2015. They were selected through the convenient sampling method and by following the inclusion criteria. They were randomly allocated to the experimental and control groups with 25 older adults in each group. The research instruments included SF-36 quality of life and demographic questionnaires. The SF-36 questionnaire was implemented before, 1 month, and 3 months after receiving the services in both groups. The data were analyzed using SPSS-20. Data analysis was performed using 1-way ANOVA with repeated measures.

Results In this study, the mean age of the control and experimental groups were 66.43 and 68.1 years, respectively. T-test showed that there was no significant difference between the control and experimental groups with regard to their ages. Furthermore, there were no significant differences between 2 groups with regard to sex, education degrees, and history of hospitalization. The mean scores of quality of life in the intervention group at baseline, 1 month and 3 months after the study were 43.95, 56.30, and 74.38, respectively. The values for the same parameters in the control group were $44.74,49.79$, and 57.82 , respectively. The lowest score in the quality of life questionnaire was 24.72 , and the highest was 86.11 . In addition, there was no significant difference between 2 groups with regard to mean scores of their quality of life at baseline; however, there were significant differences for 1 and 3 months after the study (respectively, $\mathrm{P}<0.001, \mathrm{P}<0.015$ ).

Conclusion The results showed that the day-care services significantly increased the quality of lives of older people. Therefore, it is hoped that these services can be used along with other conventional services as an effective method. Furthermore, the non-pharmaceutical interventional module can be used in promoting the quality of lives of older people. Rehabilitation services designed by the Welfare Organization for use in the day-care centers for older adults have a positive impact on the elders' quality of lives.
\end{abstract}

Key words: Elderly, Rehabilitation, Quality of life, Older adult, Day care center

\footnotetext{
* Corresponding Author:

Mahshid Foroughan, PhD

Address: Iranian Research Center on Aging, University of Social Welfare and Rehabilitation Sciences, Kodakyar Ave., Daneshjo Blv., Evin, Tehran, Iran. Tel: +98 (21) 2280004

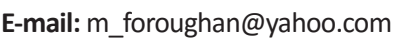




\title{
مقايسه كيفيت زندتى سالمندان قبل و بعد از دريافت بسته خدمات توانبخشى روزانه سازمان \\ sivisist.
}

\author{
محمد نصيرى'؛ "مهشيد فروغانجّ وحيد راشدى'، محمدرضا شهبازى' \\ 1- كروه مديريت توانبخشى، دانشُكَاه علوم بهزيستى و توانبخشى، تهرانه ايران.

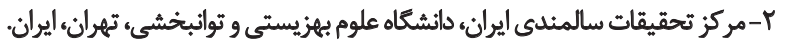

\begin{abstract}
حكبن

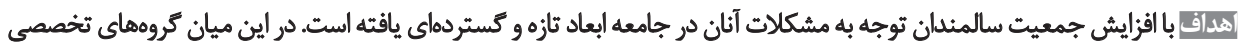

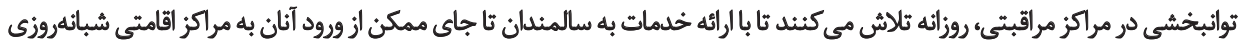

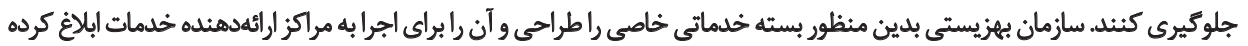

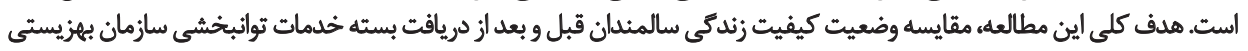

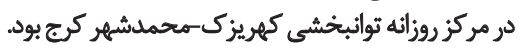

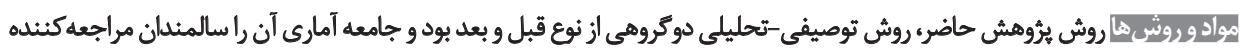

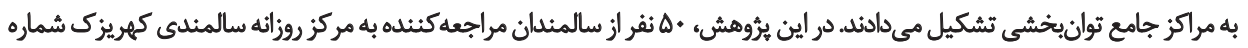

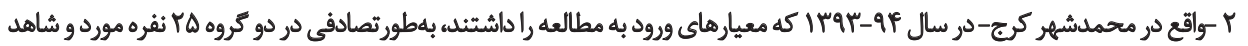

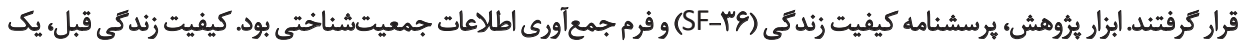

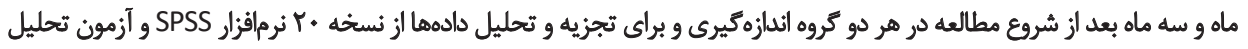

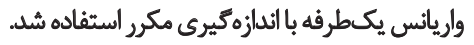

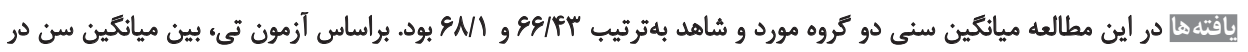

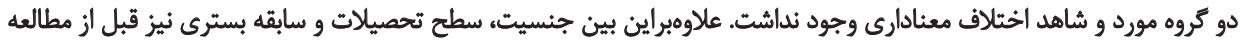

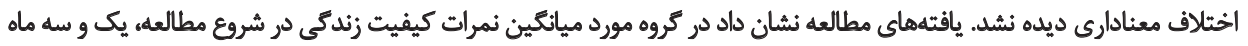

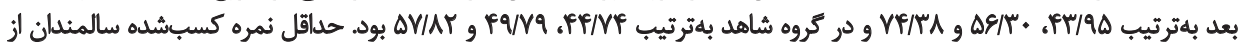

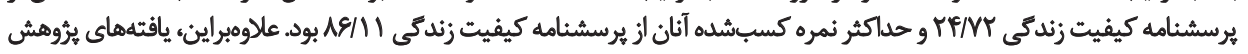

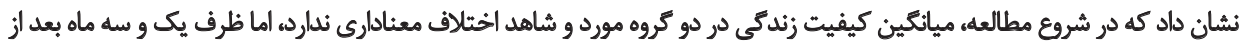

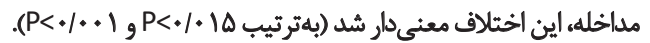

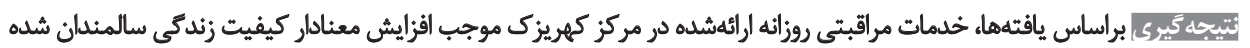

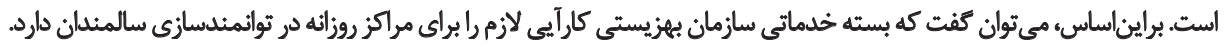

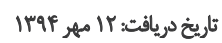

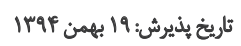

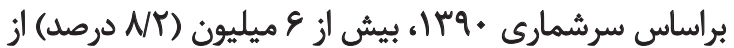

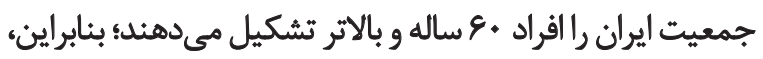

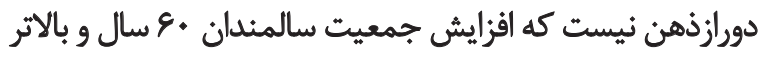

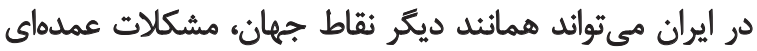

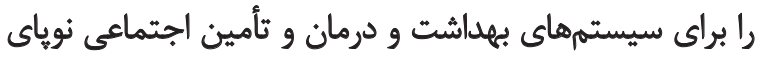

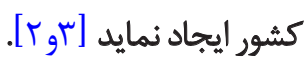

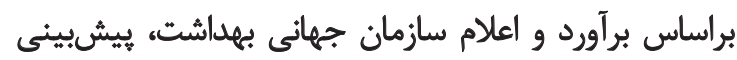

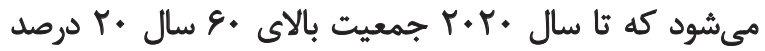

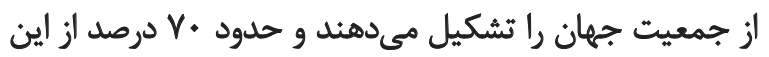

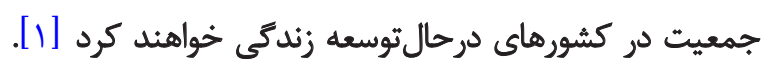

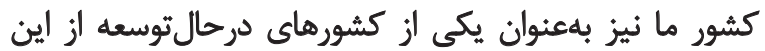
موضوع مستثنى نيست، بهطورى كه روند بيرشدن دران در كشور از 


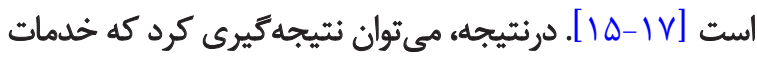

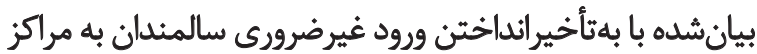

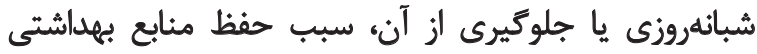

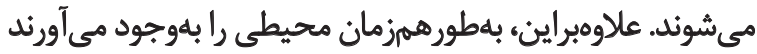

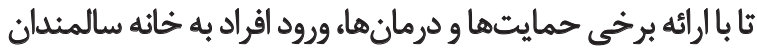

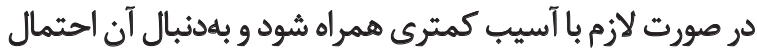

افت توان ذهنى و جسمى آنان كاهش يابد [1 ال]

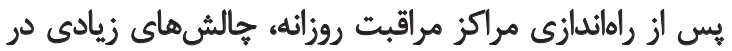

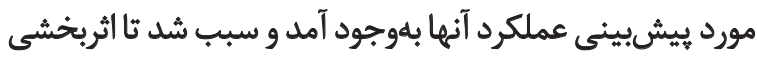

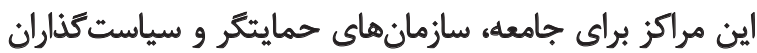

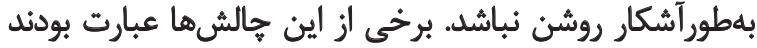

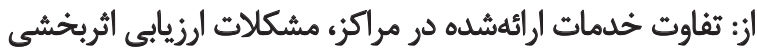

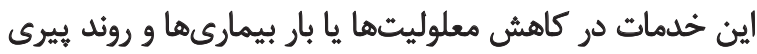

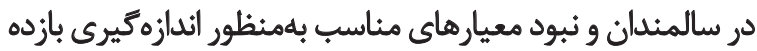

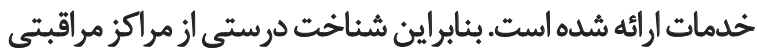

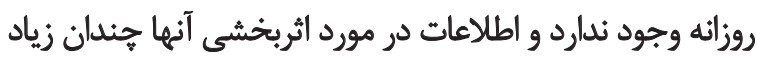
نيست

در كشور مانيز مراكز مذكور با اهداف مشابهي ثأسيس شدهانده

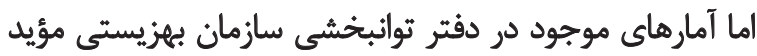

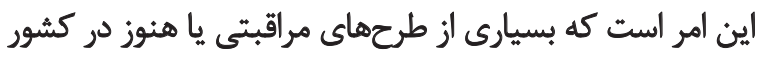

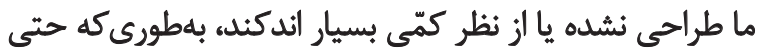

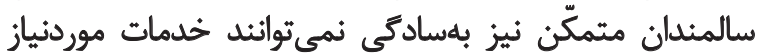

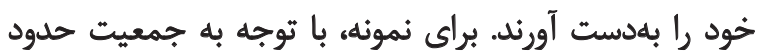

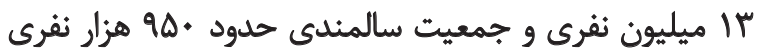

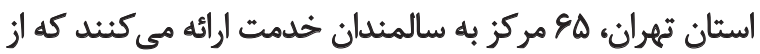

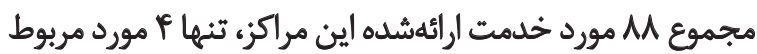

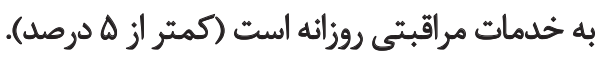

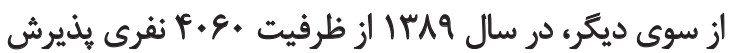

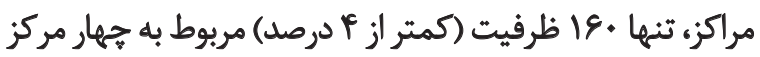

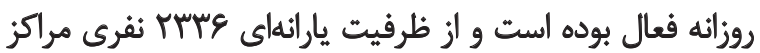

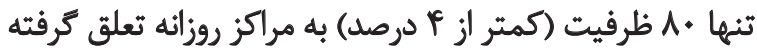

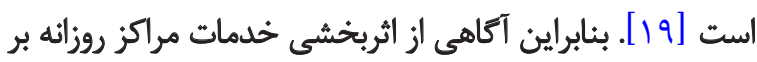

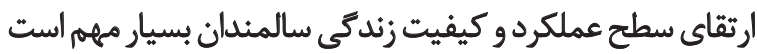

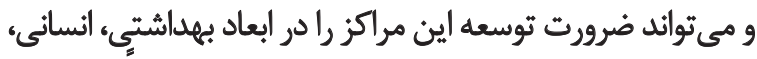

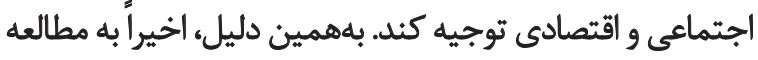

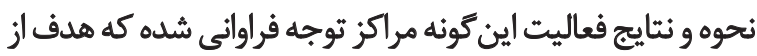

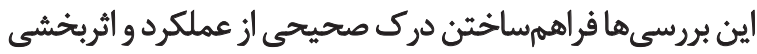
خدمات اين مراكز است.

تاكنون كارآيى برنامه توانبخشى سالمندان كه توسط سازمان

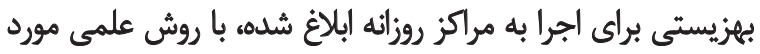

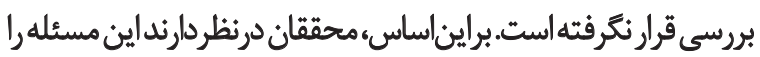

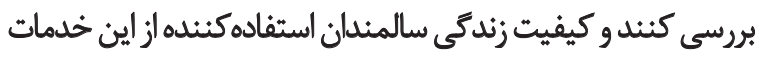

مراى ارتقاى سلامت و بيشكَيرى از بروز و ابتلا به بيمارىهاى

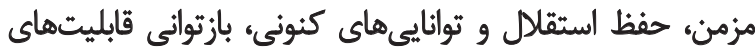

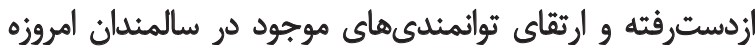

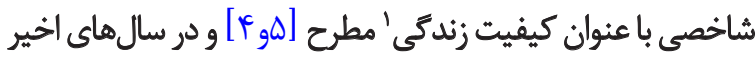

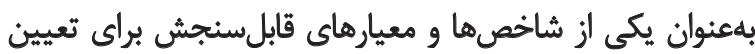

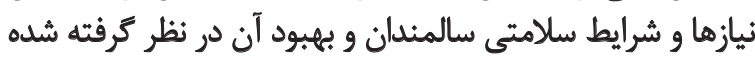

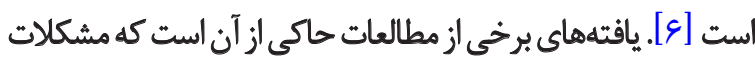

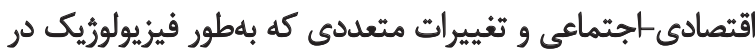

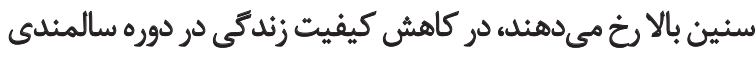

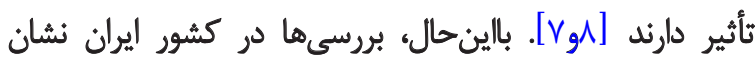

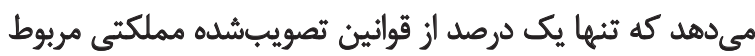

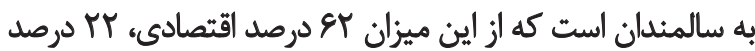

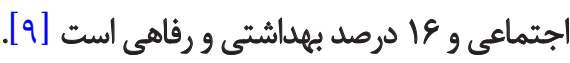

برخى مطالعات نشان مىدهد خدماتى كه با ارتقاى سطح

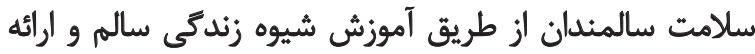

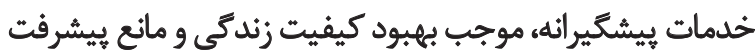

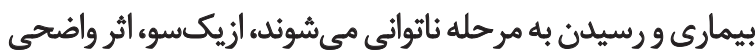

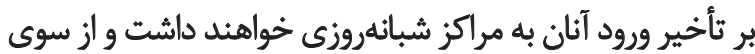

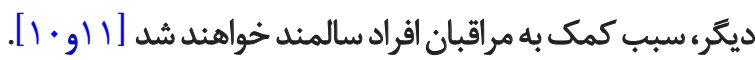

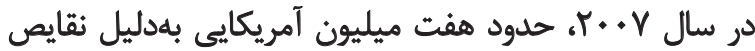

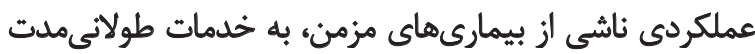

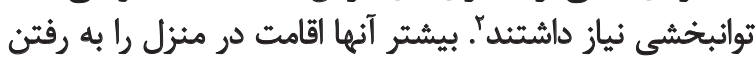

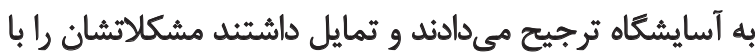

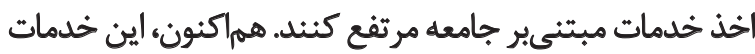

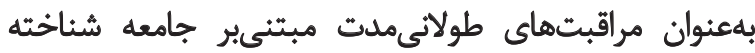

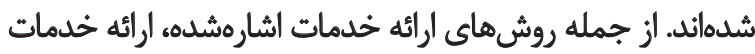

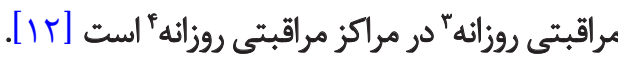

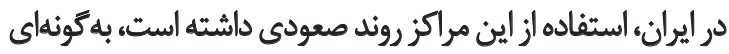

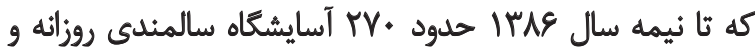

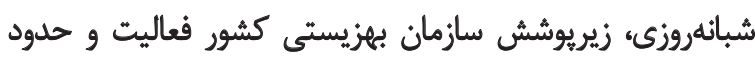

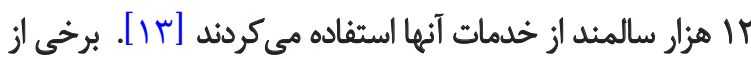

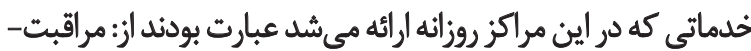

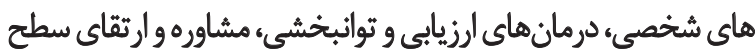

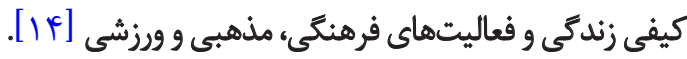
بررسى هاى متعددى نشان دادماند كه ارائه خدمات مراقبتى

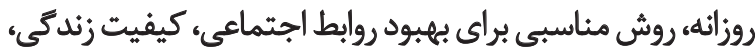

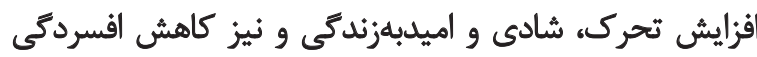

1. Quality of life

2. Long term care

3. Day care services

4. Day care centers 
هي از بيان توضيحات لازم و كسب اجازه از مسئولين مركزء افراد

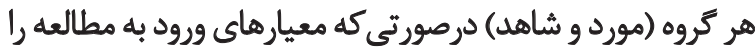

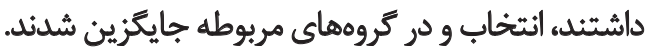

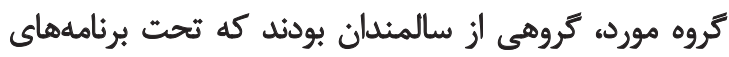

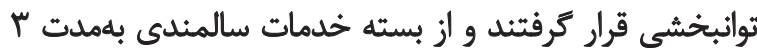

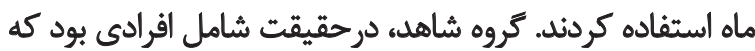

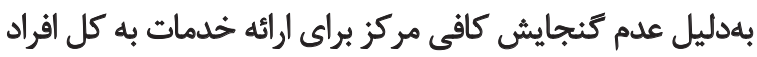

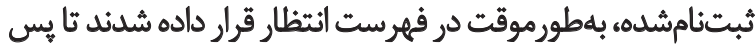

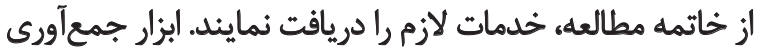

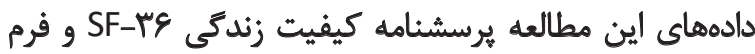
جمع آورى اطلاعات جمعيتشناختى بودي

يرسشنامه كيفيت زندگى (SF-PF) از ابزارهاى عمومى بسيار

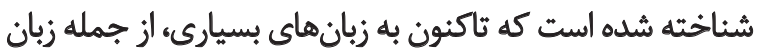

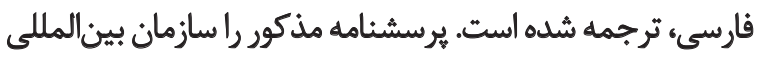

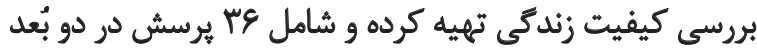

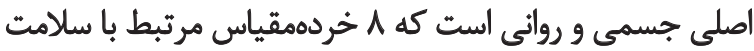

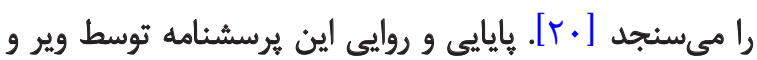

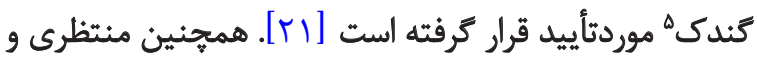

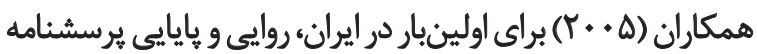

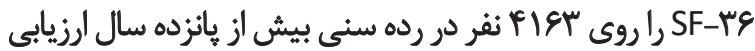

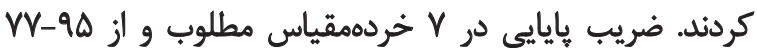

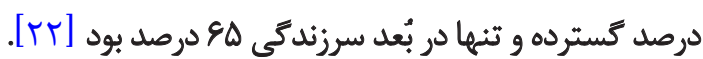

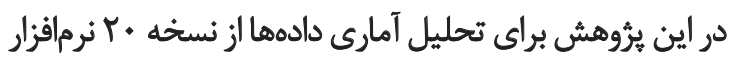

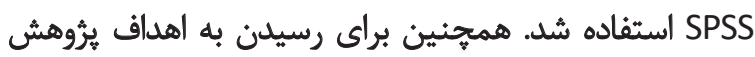

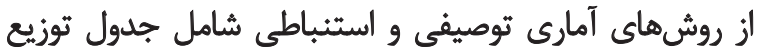

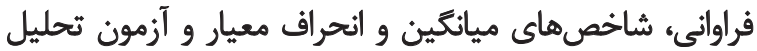

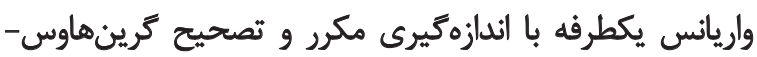

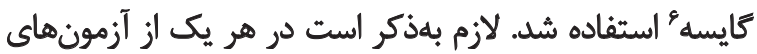

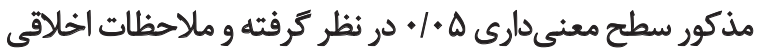

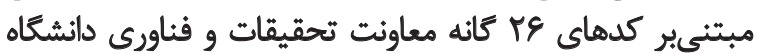

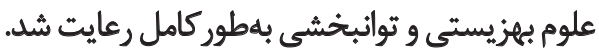

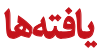

با توجه به جدول شماره ا، ميانتَين سنى در دو كروه مورد و

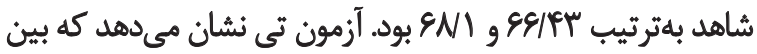

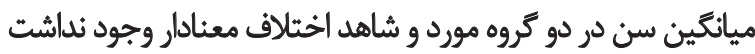

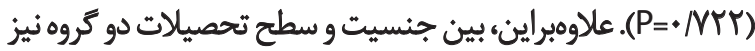
قبل از مطالعه اختلاف معناداري دين جيده نشد. براساس جدول شماره ז، ميانكين نمره كيفيت زندگى در كروه مورد

5. Ware and Gandek

6. Greenhouse-geisse

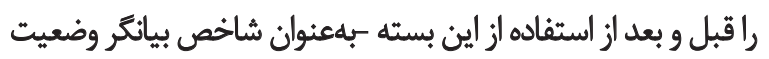

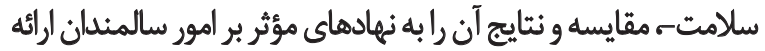
دهند تاب بهنظور برنامهريزى هائ آتى مورد استفاده قرار خيرد.

ووشي برورسي

اين يروهش از نوع توصيفى-تحليلى دوكروهى از نوع قبل و بعد

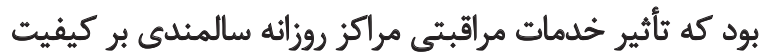

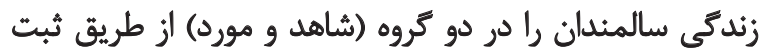

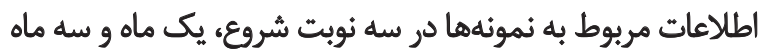

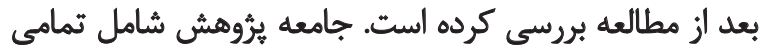

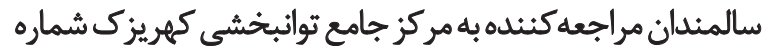

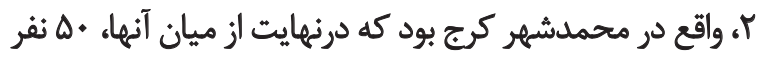

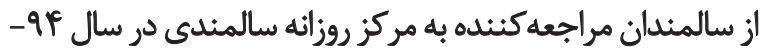

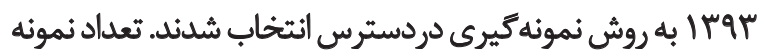

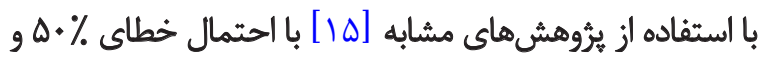

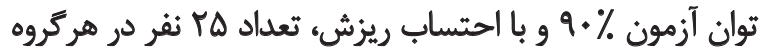
(مورد و شاهد) درنظر مرفته شد.

افرادى كه معيارهاى ورود به مطالعه را داشتند با نمونه كيرى

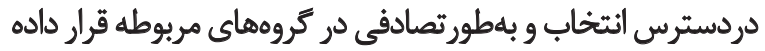

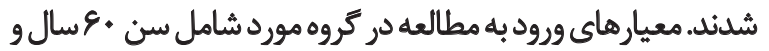

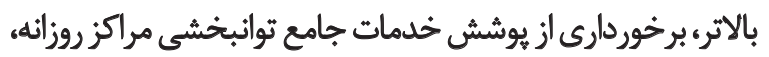

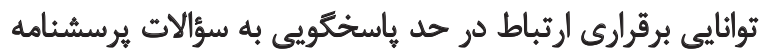

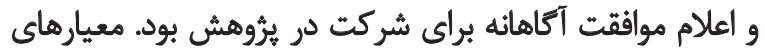

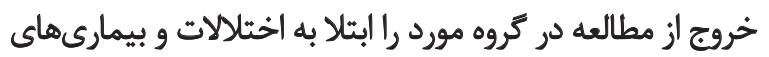

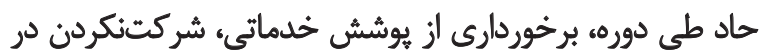
بيش از يكسوم برنامههاى توانبخشى تشكيل مي ادياد.

لازم بلهذكر است ملاكهاى ورود و خروج براى كروه شاهد

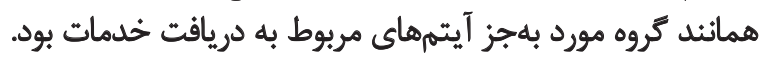

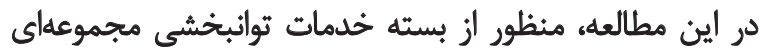
الز خدمات توانبخشى است كه دستورالعمل آن توسط سارئ سازمان

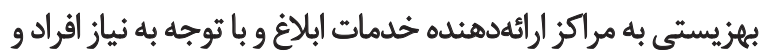
براساس تشخيص كروه توانبخشى مركز كهريزك كرج ارائه مي شيد. بخشى از خدمات مركز كه خدمت كيرندكان از آن استفاده

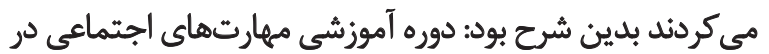

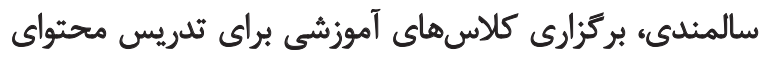

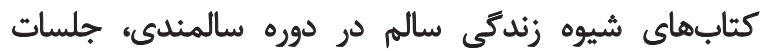

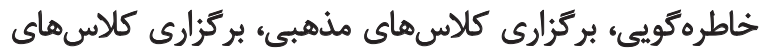

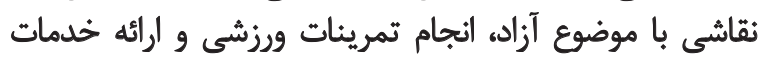

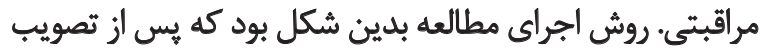

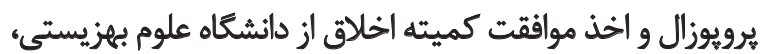

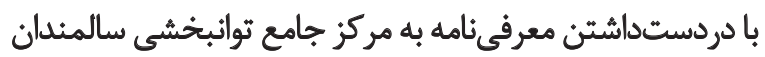

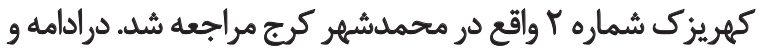


جدول ا. توزيع فراواني سن، جنس و تحصيلات سالمئدان مورد مطالعه.

\begin{tabular}{|c|c|c|c|c|c|c|c|c|c|c|}
\hline \multicolumn{4}{|c|}{ تحصيات } & \multicolumn{2}{|c|}{ جنس } & \multicolumn{3}{|c|}{ سن } & \multirow{2}{*}{ فراواني } & \\
\hline مجموع & ديبلم & سيكل & بىسواد & زن & مرد & $1+-19$ & $v+-v q$ & $8 \cdot-9 q$ & & \\
\hline ro & 1 & $\&$ & $M$ & 11 & if & $r$ & $\wedge$ & if & تعداد & \\
\hline $1 .$. & $f$ & rf & $r$ & if & $\Delta$ & ir & rT & $\Delta F$ & درصد & هورد \\
\hline ro & 1 & 8 & M & 9 & 18 & r & 1 & ir & تعداد & Aاهt \\
\hline $1 \ldots$ & r & Tr & $r$ & ק & ef & 18 & rT & ar & درصد & \\
\hline
\end{tabular}

L

جدول r. مثايسه ميائكين كيفيت زندكَى در دو كروه مورد و شاهد.

\begin{tabular}{|c|c|c|c|c|c|c|c|c|}
\hline \multicolumn{7}{|c|}{$N=\bowtie$. } & \multirow{3}{*}{\multicolumn{2}{|c|}{ متغير }} \\
\hline \multirow{2}{*}{$\mathrm{Pv}$} & \multirow{2}{*}{$d f$} & \multirow{2}{*}{$\mathrm{T}$} & \multicolumn{2}{|c|}{ شاهد } & \multicolumn{2}{|c|}{ مورد } & & \\
\hline & & & انحراف معيار & مياكيين & انحراف معيار & ميأكين & & \\
\hline.$|A| \&$ & in &.$- / M \pi$ & $1.1 \Delta V$ & 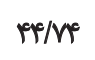 & $\mathrm{Ir/M}$ & $p \pi / q \Delta$ & شُوع مطألها & \\
\hline .1 .10 & in & $r(\Delta)$ & Q/זם & $p q / v q$ & NQA & $\Delta s / r$ & يك ماه بعد & كيفيت زندكى \\
\hline$<+1 .+1$ & p. & 8109 & V/ar & aV/Ar & $9 / n^{2}$ & ne/ra & سه ماه بعل & \\
\hline
\end{tabular}

L

دريافت خدمات روزانه به مركز مراجعه نكرده بودند. اين در حالي است كه نتايج بهدستآمده از سرشمارى عمومي

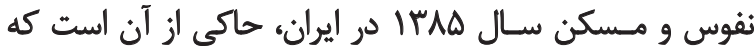

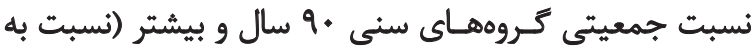

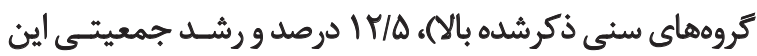

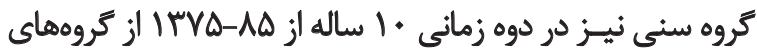

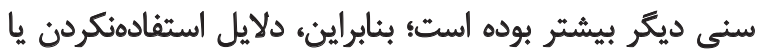

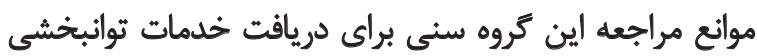

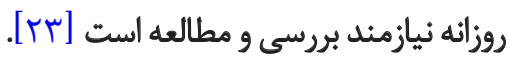

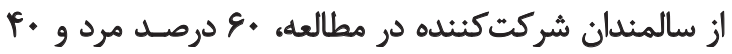

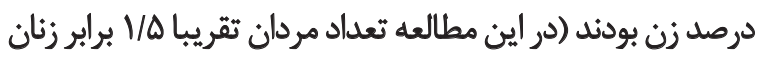

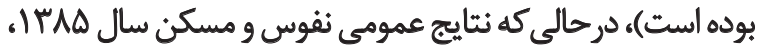

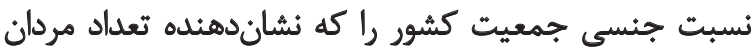

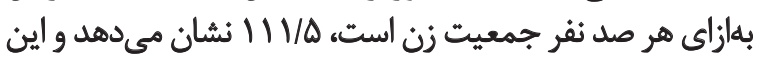

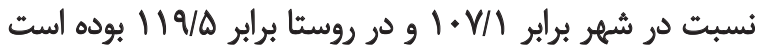

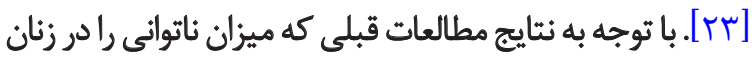

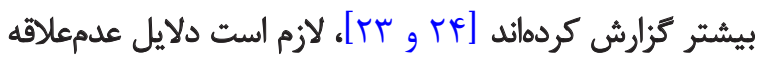

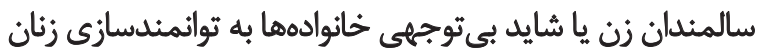
سالمند نيز موردبررسي بيشيتر قرار كيرد.

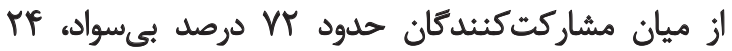

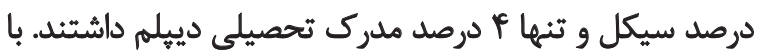

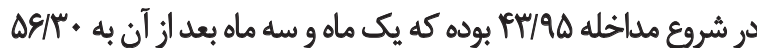

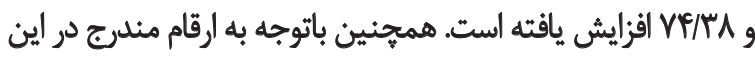

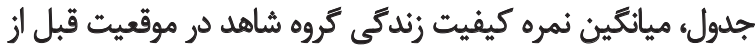

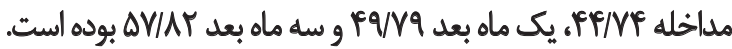
همانطوركه هشاهده مي شود نمره كيفيت زندكى سالمندان در كروه

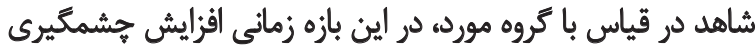

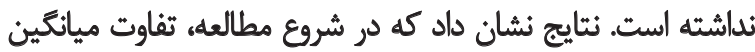

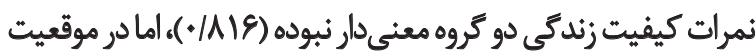

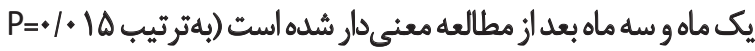

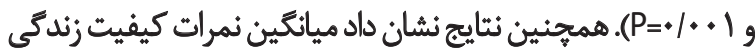

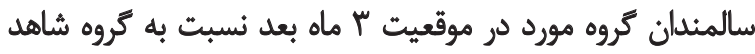

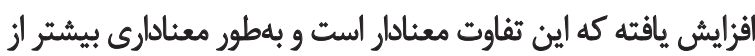

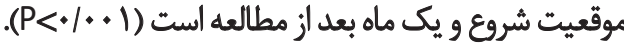

ث0!

اين يرؤهش با هدف مقايسه وضعيت كيفيت زندكى سالمندان

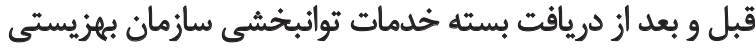

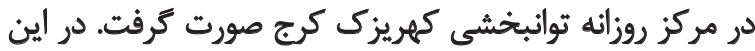

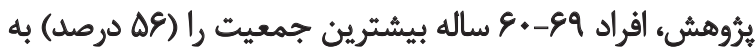
خود اختصاص داده بودند. تروه و

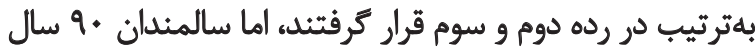

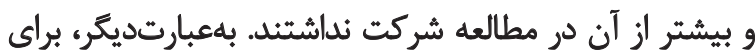


بافزيستى را دريافت كرده بودئل، هحدوديتهاي جسمى تقليل

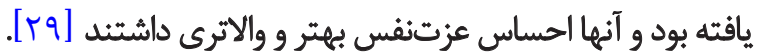

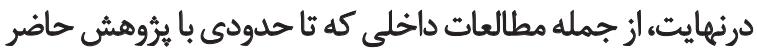

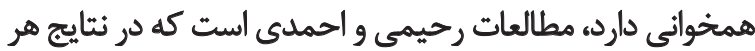

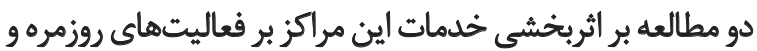

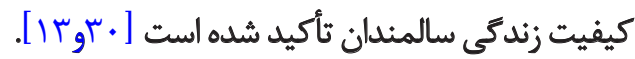

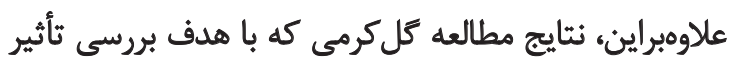

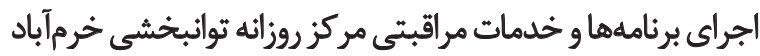

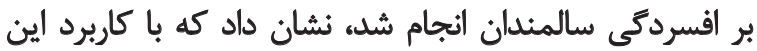

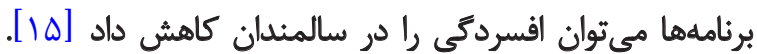

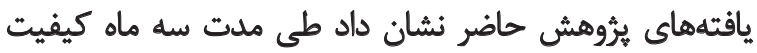

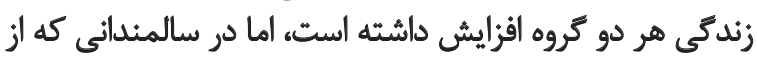

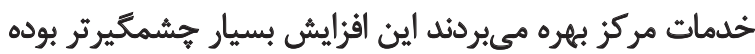

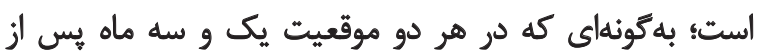
مطالعه، افزايش ميانكين كيفيت زندكى معناديت ديار است نتايج نشان داد كه بسته خدماتى مراكز روزانه در كوتامدات

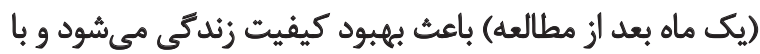

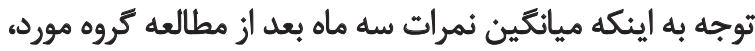

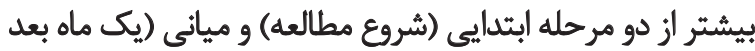

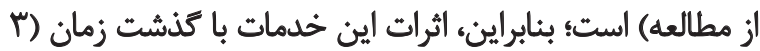

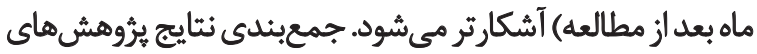

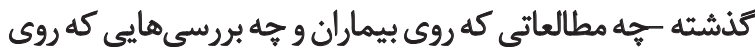

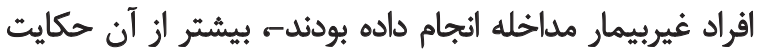

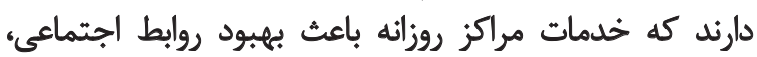

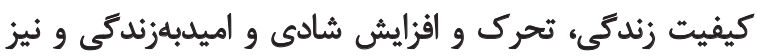

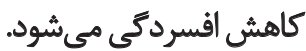

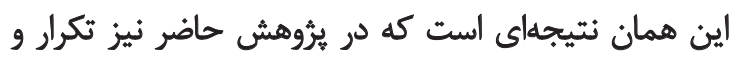

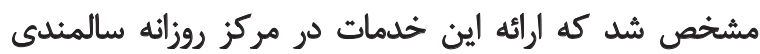

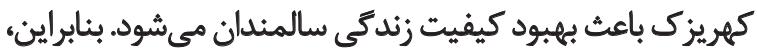

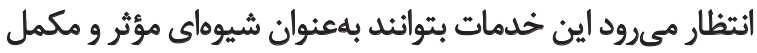

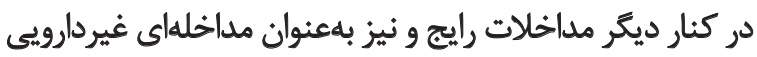

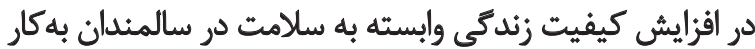

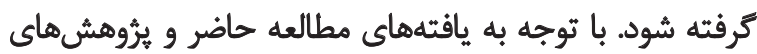

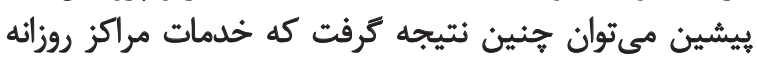

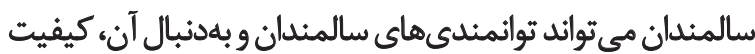

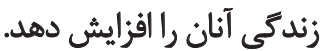

\section{نتيجهئيرى نهايى}

يافتههاى ثرؤهش حاضر، ضمن ثأييد فرضيه تحقيق نشان

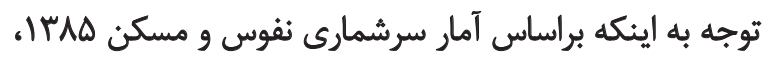

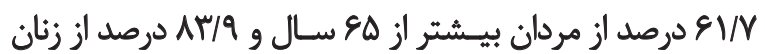

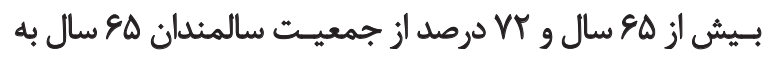

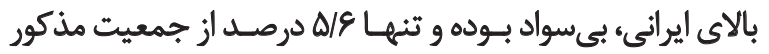

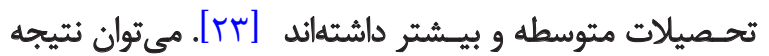

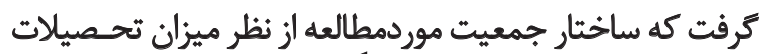

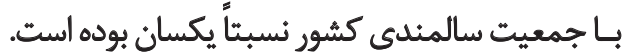
نتايج جدول شماره Y، نشان مى دهد كه بسته خدماتى ذكرشده

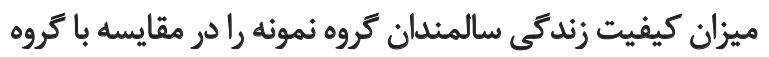

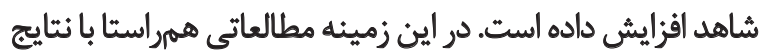

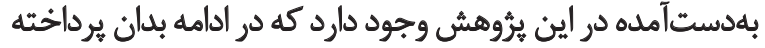

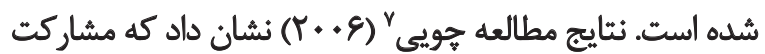

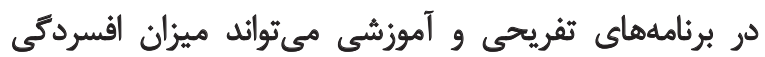

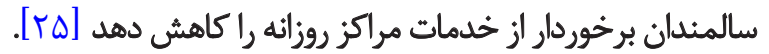

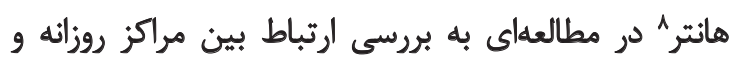

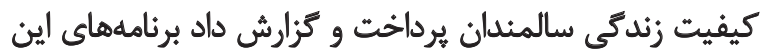

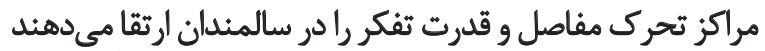

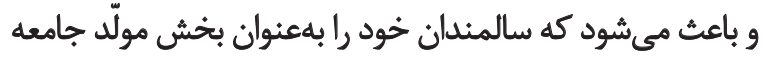

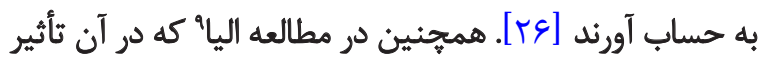

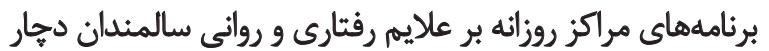

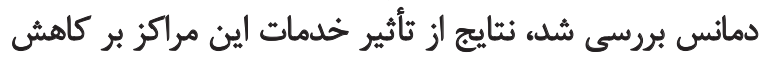

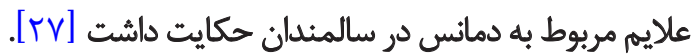

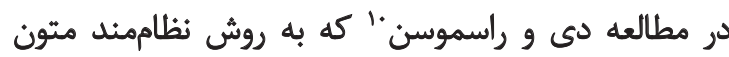

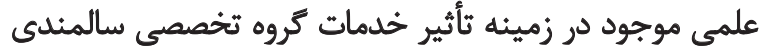

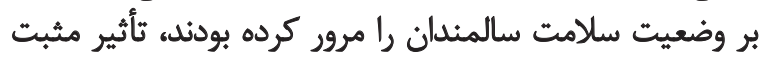

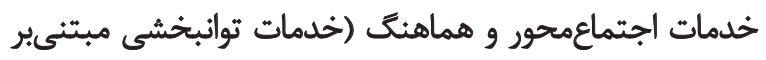

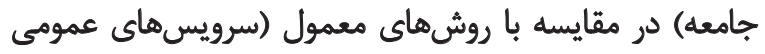

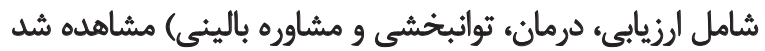

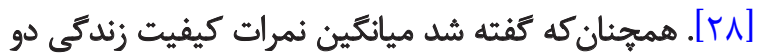

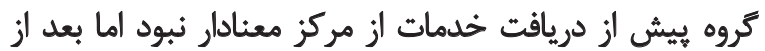

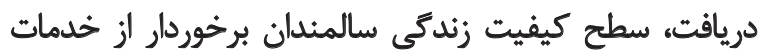

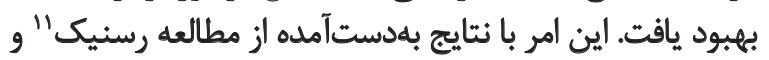

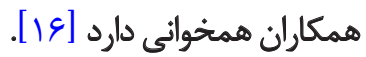
از ديكّر برُوهش هايى كه تا حدودى با مطالعه حاضر همخواني

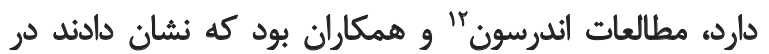

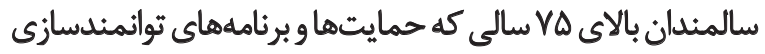




\section{References}

[1] Khazaiy K. [Psychology of aging and the role of the role of social support (Persian)]. In: Mahatir M, editor. Ageing Issues in Iran and the World. Tehran: Ketab-e Ashna; 2002, pp. 363-371.

[2] Momeni KH, Karimi H. [Comparison of mental health between elderly admitted in sanitarium with elderly in sited in personal home (Persian)]. Behbood Journal. 2010; 14(4):328-35.

[3] Rashedi V, Gharib M, Yazdani AA. Social participation and mental health among older adults in Iran. Iranian Rehabilitation Journal. 2014; 12(19):9-13.

[4] Rimaz S, Abolghasemi J, Seraji S. [The relationship of different dimensions of social support with older adults' quality of life in the $8^{\text {th }}$ district of Tehran in 2013 (Persian)]. Journal of Education and Community Health. 2015; 2(1):32-9.

[5] Nabavi S, Shoja M, Mohammadi S, Rashedi V. [Health-related quality of life in community-dwelling older adults of Bojnourd (Persian)]. Journal of North Khorasan University of Medical Sciences. 2014; 6(2):433-9.

[6] Bonomi AE, Patrick DL, Bushnell DM, Martin M. Validation of the United States' version of the World Health Organization Quality of Life (WHOQOL) instrument. Journal of Clinical Epidemiology. 2000; 53(1):1-2.

[7] Afsharkohan J, koolivand SH. [Structured study of quality of the life for the elderly in Iran (2004-13) (Persian)]. Journal of Rehabilitation. 2015; 10(3):50-36

[8] Rashedi V, Gharib M, Rezaei M, Yazdani AA. [Social support and anxiety in the elderly of Hamedan, Iran (Persian)]. Journal of Rehabilitation. 2013; 14(2):110-5.

[9] Farzianpour F, Tajvar M. [Elderly health and a review on different aspects of their life (Persian)]. Tehran: Nasle Farda and Arjmand Press; 2004.

[10] Mohtasham AZ, Farazmand A, Toloei M. [Causes of patients' hospitalization in Guilan university hospitals (Persian)]. Journal of Guilan University of Medical Sciences. 2002; 11(42):28-32.

[11] O'Keefe J, Siebenaler K. Adult day services: A key community service for older adults. New York: Department of Health and Human Services; 2006.

[12] Halter J, Ouslander J, Tinetti M, Studenski S, High K, Asthana S. Hazzard's geriatric medicine and gerontology. Oxford: McGrawHill Publication; 2009.

[13] Rahimi M, Fadayevatan R, Davatgaran R. [Effectiveness of Day Care Servies of Karaj Kahrizac Rehabilitation Center on Activities of Daily Living among Older People (Persian)]. Iranian Journal of Ageing. 2011; 8(3):7-16.

[14] Hashemnejad H, Boelvardi S. Taking advantage of the daily residential space for improving the quality of elderly's life. Journal of Architecture and Urban Utopia. 2009; 2:75-90

[15] Gol Karami S, Mobaraki H, Kamali M, Farhodi F. [The effect of empowerment programs on geriatric depression in daily rehabilitation Farzanegan center of khorramabad city (Persian)]. Modern Rehabilitation. 2013; 6(4):65-70.

[16] Resnick B, Luisi D, Vogel A. Testing the Senior Exercise Selfefficacy Project (SESEP) for Use with Urban Dwelling Minority Older Adults. Public Health Nursing. 2008; 25(3):221-34.
داد كه خدمات مراكز روزائه در قالب بسئه خدماتي سازمان

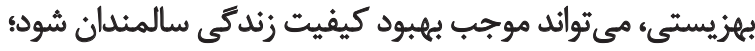

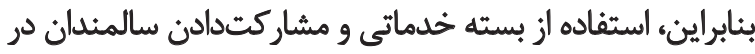

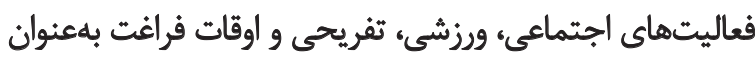

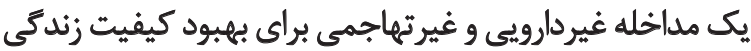

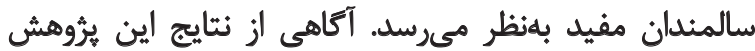

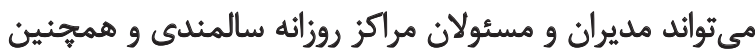

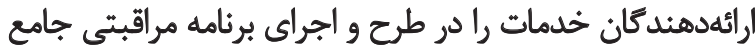

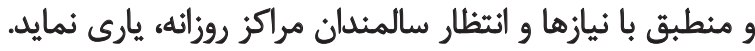

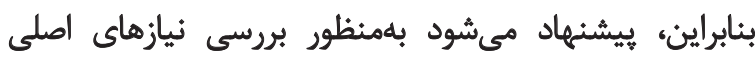

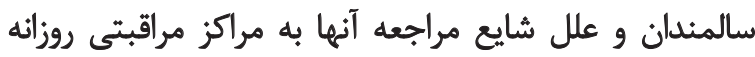

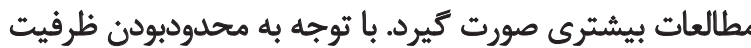

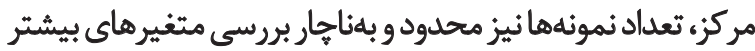

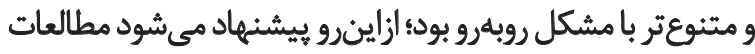

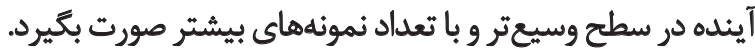

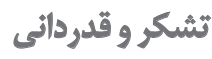

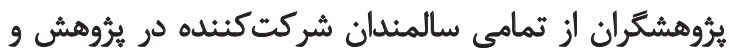

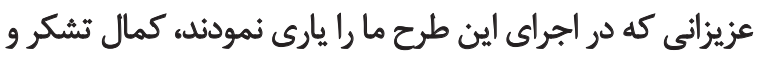
قدرداني هي نمئيند. 
[17] Rashedi V, Rezaei M, Gharib M, Nabavi S. [Social support for the elderly: Comparison between home and nursing home (Persian)]. Journal of North Khorasan University of Medical Sciences. 2013; 5(2):351-6.

[18] Alteras T. Adult day health care services: Serving the chronic health needs of frail elderly through cost-effective, non-institutional care. Health Management Associates. 2007; 23:6.

[19] Adib-Hajbaghery M, Rajaei M. [Lived experiences of elderly home residents: A qualitative study (Persian)]. Journal of Kermanshah University of Medical Sciences. 2011; 15(5):372-383.

[20] Manokian A, Pedram Razi S, Monjamed Z, Faghihzadeh S. [Comparison between oncology and labor delivery nurse's job satisfaction (Persian)]. Hayat. 2007; 13(3):49-55.

[21] Ware JE, Gandek B. Overview of the SF-36 health survey and the international quality of life assessment (IQOLA) project. Journal of Clinical Epidemiology. 1998; 51(11):903-12.

[22] Montazeri A, Goshtasebi A, Vahdaninia M, Gandek B. The short form health survey (SF-36): Translation and validation study of the Iranian version. Quality of Life Research. 2005; 14(3):875-82.

[23] Statistical center of Iran. Findings of 2006 national population and housing census. Presidency of the I.R.I, Vice presidency for strategic planning and supervision. Tehran: Statistical center of Iran; 2007

[24] Nodehimogadam A, Ehsanifar F. [The Survey Abnormal Physicalmotor Elderly Respect to Center of Rehabilitation Organization Walfare of Tehran (Persian)]. Iranian Journal of Ageing. 2006; 1(2):125-131

[25] Chui KC. Participating leisure and recreational activities and depressive symptoms among Chinese elder people residing in institutions [PhD thesis]. Hong Kong: The University of Hong Kong; 2006.

[26] Hunter S. Adult day care: Promoting quality of life for the elderly. Journal of Gerontological Nursing. 1992; 18(2):17-20.

[27] Femia EE, Zarit SH, Stephens MAP, Greene R. Impact of adult day services on behavioral and psychological symptoms of dementia. Gerontologist. 2007; 47(6):775-88.

[28] Day P, Rasmussen P. What is the evidence for the effectiveness of specialist geriatric services in acute, post-acute and sub-acute settings. New Zealand Health Technology Assessment. 2004; 7(3):1-149.

[29] Andersson M, Hallberg IR, Edberg AK. Old people receiving municipal care, their experiences of what constitutes a good life in the last phase of life: A qualitative study. International Journal of Nursing Studies. 2008; 45(6):818-28.

[30] Ayubi A. [The Effect of Aging Health Care Package Center of Vitality Elderly Yazd on Quality of Lifeand Activities of daily Living Elderly women (Persian)] [PhD thesis]. Tehran: University of Social Welfare and Rehabilitation Sciences; 2013. 